THE AstrophysiCal Journal, 346:756-762, 1989 November 15

(C) 1989. The American Astronomical Society. All rights reserved. Printed in U.S.A.

\title{
VLA OBSERVATIONS OF AMMONIA AND CONTINUUM IN REGIONS WITH HIGH-VELOCITY GASEOUS OUTFLOWS. II.
}

\author{
José M. TORRELlES \\ Instituto de Astrofisica de Andalucía, CSIC \\ Paul T. P. Ho and Luis F. Rodríguez ${ }^{1}$ \\ Harvard-Smithsonian Center for Astrophysics \\ JoRge CANTÓ \\ Instituto de Astronomía, UNAM \\ AND \\ LOURDES VERDES-MONTENEGRO \\ Instituto de Astrofisica de Andalucía, CSIC \\ Received 1989 January 30; accepted 1989 May 17
}

\begin{abstract}
We present VLA observations of the $(1,1)$ inversion transition of ammonia toward four regions with highvelocity gaseous outflow: HH 26-IR, GGD 12-15, Serpens, and V645 Cygni. We detected small-scale structure $\left(\sim 10^{\prime \prime}\right)$ in all sources. In HH 26-IR and GGD 12-15 we find double-maximum structures that could be related to the collimation processes of the outflow. The condensations observed in Serpens and V645 Cygni appear to be the cores of the more extended structures mapped with a single dish.

Subject headings: interstellar: molecules - nebulae: $\mathrm{H}$ II regions
\end{abstract}

\section{INTRODUCTION}

The discovery of the molecular outflow phenomenon by Zuckerman, Kuiper, and Rodriguez Kuiper (1976), and Kwan and Scoville (1976), and the detection of the bipolar geometry that characterizes most of the outflows (Snell, Loren, and Plambeck 1980; Rodríguez, Ho, and Moran 1980), were followed in subsequent years by the detection of many other examples (Rodríguez et al. 1982; Bally and Lada 1983). These studies established observationally that the earliest stages of stellar evolution are associated with strong mass outflows. It is now well established that a large fraction of the molecular outflows appear as bipolar (see, e.g., Lada 1985 for a review) and that they are associated with high-density $\left[n\left(H_{2}\right) \geq 10^{4}\right.$ $\left.\mathrm{cm}^{-3}\right]$ molecular cores that often engulf the suspected exciting sources of the outflows (Torrelles et al. 1983, 1986a; Schwartz, Waak, and Smith 1983; Heyer et al. 1986; Myers et al. 1988). In the most conspicuous bipolar outflow sources the molecular cores sometimes have elongated shapes, with the major axis aligned perpendicular to the outflow axis, suggesting a possible role in the collimation processes (Torrelles et al. 1983; Schwartz, Waak, and Smith 1983; Rodríguez 1988 for a review). In a number of cases, optical jets are also found to emanate from young objects embedded in the molecular clouds, implying that collimation processes already operate at circumstellar size scales (see, e.g., Mundt 1986 for a review). At present it is unclear that such jets have sufficient momentum and energy to drive the large-scale molecular outflows (see, e.g., Sarcander, Neckel, and Elsässer 1985). Thus, to determine the scale and structure of the focusing mechanism of outflows, observations of high-density gas must be sensitive to a large dynamic range of physical size scales around the exciting sources. In the past few years we have extended our single-dish $\mathrm{NH}_{3}$ studies (with typical resolution of 1.5) with interferometric $\mathrm{NH}_{3}$ studies (with typical resolution of $5^{\prime \prime}-10^{\prime \prime}$ ) using the Very Large Array (VLA) of the National Radio Astronomy Observatory (NRAO). ${ }^{2}$ These $\mathrm{VLA}-\mathrm{NH}_{3}$ studies have been useful in (1) Defining the morphologies of the small-scale molecular structures at the centers of the outflow regions; (2) identifying the location of the exciting source; (3) determining the energetic effect of the outflow on the high-density gas in terms of heating and turbulence; and (4) measuring the velocity field of the molecular gas. We find that the high angular resolution provided by interferometry makes possible a detailed study of the effects of the star formation on the molecular cores. Sources studied by our group include L1551, HH 26-IR, GGD 12-15, Serpens, V645 Cygni, S140, and Cepheus A (Torrelles et al. 1985b [Paper I], hereafter THRC; 1986b), AFGL 2591 (Torrelles et al. 1989a), Monoceros R2 (Torrelles et al. 1989b), and NGC 2024 (Ori B; Ho et al. 1989). Other outflow sources studied with the VLA using the $\mathrm{NH}_{3}$ lines include W51 (Ho, Genzel, and Das 1983), G35.2-0.74 (Little et al. 1985), Orion A (Genzel et al. 1982; Pauls et al. 1983), HH 7-11 (Rudolph 1989).

In 1982 we observed and reported (THRC) nondetections toward HH 26-IR, GGD 12-15, Serpens, and V645 Cygni. The analysis at the time was based on averages of several spectral channels in order to improve sensitivity. We have now reanalyzed those data. We find that emission is detected with the VLA in all four sources. The emission is confined, however, to a single spectral channel, except for GGD 12-15, where ammonia emission is present at two velocity components. In $\S$ II we explain the new data reduction, in $\S$ III we discuss the individual sources, and in $\S$ IV we give some general comments and conclusions on our results.

\section{DATA ANALYSIS}

The observations were made with the VLA in the D configuration during 1982 November 13-15. The details of the obser-

\footnotetext{
${ }^{1}$ On sabbatical leave from Instituto de Astronomía, UNAM.

${ }^{2}$ NRAO is operated by Associated Universities, Inc., under contract with the National Science Foundation.
} 
vations are given in THRC. Individual spectral channel maps have been obtained by Fourier transformation of the calibrated visibility data. The spectral resolution is $97.7 \mathrm{kHz}(1.2$ $\left.\mathrm{km} \mathrm{s}^{-1}\right)$. We made a series of maps by convolving the $(u, v)$ data with Gaussian tapers (half-width at 30\%) ranging from 6 to $60 \mathrm{k} \lambda$ with natural weighting. For the four sources analyzed here, the optimal angular resolution turns out to be $\sim 10^{\prime \prime}-15^{\prime \prime}$. We detect $\mathrm{NH}_{3}(1,1)$ emission in all of the reanalyzed sources. In each case, the ammonia emission was detected essentially in a single spectral channel. We present here CLEANed (Clark 1980) maps. Primary-beam corrections were not applied. In GGD 12-15 the average of the line-free channels has been subtracted to remove the continuum emission from the $\mathrm{H}$ II region located at $\alpha(1950)=06^{\mathrm{h}} 08^{\mathrm{m}} 24^{\mathrm{s}} 1, \delta(1950)=$ $-06^{\circ} 11^{\prime} 08^{\prime \prime}$. This source has a flux $S_{v} \simeq 80 \mathrm{mJy}$ at $1.3 \mathrm{~cm}$ (Rodríguez et al. 1980; THRC).

\section{INDIVIDUAL SOURCES}

\section{a) $\mathrm{HH} 26-\mathrm{IR}$}

In Figure 1 we show the $10 \mathrm{k} \lambda$ Gaussian tapered map (synthesized beam of $\simeq 15^{\prime \prime}$ ) of the main component of the $(1,1)$ ammonia transition at $V_{\mathrm{LSR}}=10.3 \mathrm{~km} \mathrm{~s}^{-1}$. We detected an ammonia condensation with two maxima oriented with a position angle (p.a.) of $\simeq 26^{\circ}$. The south ammonia peak has a flux of $\sim 380$ mJy beam $^{-1}$, while the north peak flux is $\sim 210 \mathrm{mJy}$ beam $^{-1}$. However, this northern peak is located $\sim 50^{\prime \prime}$ northeast of the phase center. Correction for primary-beam attenuation would result in a flux of $340 \mathrm{mJy}^{\text {beam }}{ }^{-1}$, similar to the southern peak. Relatively weak ammonia emission at the $3 \sigma$ level ( $1 \sigma=38 \mathrm{mJy})$ joins the two maxima. We estimate for the overall structure a size of $\sim 55^{\prime \prime} \times 30^{\prime \prime}(\sim 0.12 \mathrm{pc} \times 0.07 \mathrm{pc}$, at a distance of $460 \mathrm{pc}$; Herbig 1966). Herbig-Haro objects 25 and 26 (positions adopted from Strom et al. 1986) are located at the border of each of the ammonia maxima. The infrared source SSV 59 (Strom, Strom, and Vrba 1976; Cohen and Schwartz 1983), also detected at the IRAS bands and suggested to be the exciting source of $\mathrm{HH} 25$ and $\mathrm{HH} 26$ as well as of the bipolar molecular outflow of the region (Snell and Edwards 1982), lies between $\mathrm{HH} 25$ and $\mathrm{HH}$ 26, but is closer to the southern ammonia peak. Another IRAS source (see Fig. 1) seems closer to the northern ammonia peak. However, given the positional errors, this northern $I R A S$ source may be located midway of the two ammonia maxima.

We must also note that Matthews and Little (1983) predicted in HH 26-IR the presence of an optically thick clump of dimension $1^{\prime} \times 0.25$, very similar to what we have in fact observed. Recently, a similar ammonia structure was also observed by Menten, Walmsley, and Mauersberger (1987) by using the Effelsberg $100 \mathrm{~m}$ telescope.

In Figure 2 we show a spatial velocity diagram along an axis with p.a. $=26^{\circ}$ through the position of the two ammonia peaks. No significant ammonia emission $(\leq 4 \sigma)$ is detected at the channels adjacent to $V_{\mathrm{LSR}}=10.3 \mathrm{~km} \mathrm{~s}^{-1}$. No velocity shift is observed between the northern and southern ammonia peaks within our spectral resolution of $1.2 \mathrm{~km} \mathrm{~s}^{-1}$. This diagram clearly shows the inner satellite hyperfine lines $(1,1$; is) at $V_{\mathrm{LSR}} \sim 3$ and $18 \mathrm{~km} \mathrm{~s}^{-1}$. In Figure 3 we show the inner satellite hyperfine line to main hyperfine line flux ratio (gray scale) superposed on the contour map of the main hyperfine component. To compute these ratios, we clipped the individual maps at the $3 \sigma$ level. A flux ratio of 0.6 is obtained at the position of both ammonia peaks. This value implies an opacity $\tau(1,1 ; \mathrm{m}) \simeq 3$ (see, e.g., Ho and Townes 1983). Assuming LTE for the rotational states of ammonia at $T_{R}=T_{K}=15 \mathrm{~K}$ (from CO data; White and Phillips 1981), an abundance $\left[\mathrm{NH}_{3} / \mathrm{H}_{2}\right]=10^{-8}$ (Herbst and Klemperer 1973) and a line width $\Delta V=1.2 \mathrm{~km} \mathrm{~s}^{-1}$ (upper limit), we estimate a hydrogen column density $N\left(\mathrm{H}_{2}\right) \simeq 2 \times 10^{23} \mathrm{~cm}^{-2}$ and a mass $\simeq 27 M_{\odot}$ for the overall VLA-ammonia structure. This hydrogen column density implies a visual extinction $A_{V} \simeq 200 \mathrm{mag}$ (Spitzer 1978).

Torrelles et al. (1983), by single-dish ammonia observations, detected an elongated high-density molecular gas structure with size $\sim 0.8 \mathrm{pc} \times 0.3 \mathrm{pc}$ oriented perpendicular to the $\mathrm{CO}$ bipolar outflow (Snell and Edwards 1982). This orientation, together with an observed velocity gradient of $\sim 0.5 \mathrm{~km} \mathrm{~s}^{-1}$ $\mathrm{pc}^{-1}$ along the major axis, suggested an interpretation in terms of an interstellar toroid that may collimate the bipolar outflow.

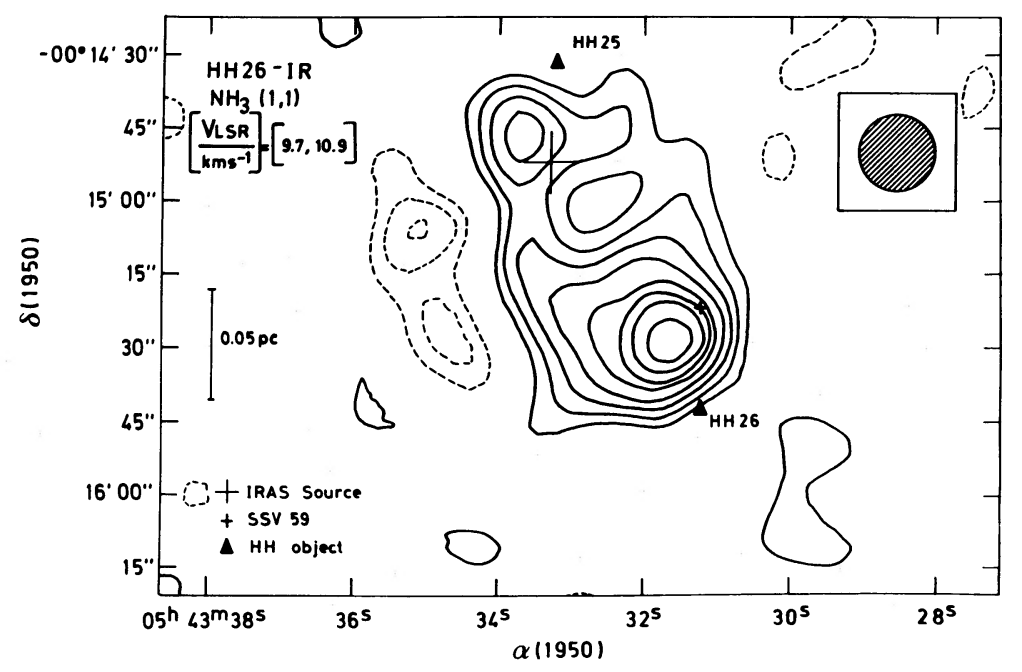

Fig. 1. - Contour map of the $(1,1)$ ammonia main transition in HH $26-\mathrm{IR}$ at the $\left(V_{\mathrm{LSR}} / \mathrm{km} \mathrm{s}^{-1}\right)=(9.7,10.9)$ range of velocity. Synthesized beam size is $15^{\prime \prime}$. Contour levels are $(-5,-4,-3,-2,2,3,4,5,6,7,8,9) \times 38 \mathrm{mJy}_{\text {beam }}^{-1}(\mathrm{rms}=38 \mathrm{mJy})$. Other objects are indicated in the figure. Positional error of the northern IRAS source is $\sim \pm 30^{\prime \prime}$. 


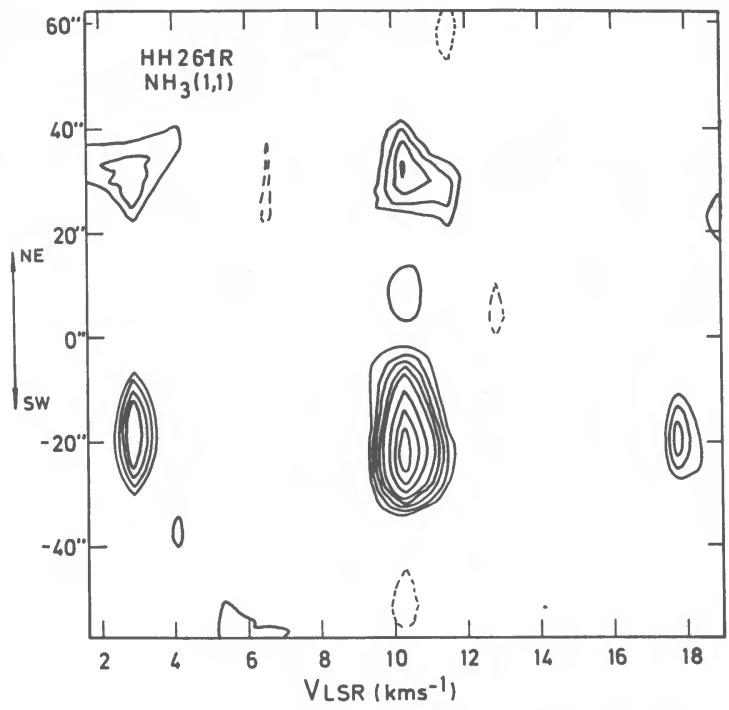

FIG. 2.-Spatial velocity diagram along an axis through the positions of the two ammonia peaks (p.a. $=26^{\circ}$ ) of Fig. 1 . Synthesized beam is $15^{\prime \prime}$. Contour levels are as in Fig. 1. Spatial coordinate (vertical axis) is with respect to $\alpha(1950)=05^{\mathrm{h}} 43^{\mathrm{m}} 32^{\mathrm{s}} .6$ and $\delta(1950)=-00^{\circ} 15^{\prime} 10^{\prime \prime}$. Note the detection of the inner satellite hyperfine lines $(1,1 ;$ is $)$ at $V_{\mathrm{LSR}} \sim 3$ and $18 \mathrm{~km} \mathrm{~s}^{-1}$.

In Figure 4 we show an overlay of the single-dish $\mathrm{NH}_{3}$ map, the $\mathrm{CO}$ bipolar outflow, and the VLA- $\mathrm{NH}_{3}(1,1)$ map. We find that the VLA and single-dish $\mathrm{NH}_{3}(1,1)$ structures are elongated in the same direction despite a difference in size scale of $\sim 7$ times. The $\mathrm{VLA}-\mathrm{NH}_{3}(1,1)$ maxima may represent the inner parts of the interstellar toroid proposed by Torrelles et al. (1983). This morphology would be consistent with the fact that both peaks have similar fluxes and opacities (see above). In addition, it appears that this inner cavity, with a radius
$R \sim 0.06 \mathrm{pc}$, can be produced by the pressure of the stellar wind associated with the powering source of the $\mathrm{CO}$ outflow. Assuming pressure equilibrium at a radius $R$ between the stellar wind and the molecular gas, with volume mass density $\rho=2 m_{h} n\left(\mathrm{H}_{2}\right)$, and velocity dispersion in one direction $\sigma_{r}$, we have $\dot{M} V_{*} / 4 \pi R^{2}=\rho \sigma_{r}^{2}$. For a stellar wind momentum rate of $\dot{M} V_{*}=1.5 \times 10^{-3} M_{\odot} \mathrm{km} \mathrm{s}^{-1} \mathrm{yr}^{-1}$ (Snell and Edwards 1982), and adopting $R=0.06$ pc (the apparent physical separation between the two VLA-ammonia maxima), and $\sigma_{r}=1.2$ $\mathrm{km} \mathrm{s}^{-1}$ (the upper limit of the ammonia line width), we require a hydrogen volume density of $n\left(\mathrm{H}_{2}\right) \simeq 5 \times 10^{5} \mathrm{~cm}^{-3}$. This value is in fact consistent with the value derived from the hydrogen column density. This result implies that a stellar wind associated with a source located midway between the two VLA-ammonia maxima can be stopped by the high-density gas.

In this model of a centrally evacuated toroid, it is difficult to explain the excitation of $\mathrm{HH} 25$ and $\mathrm{HH} 26$ at the outermost parts of the VLA structure by a central stellar wind without special geometry such as holes within the toroid.

If the VLA-ammonia structure represents two independent clumps, with SSV 59 and/or the northern IRAS source as the exciting source(s) of the molecular outflow, a stellar wind related to these sources could interact with the ammonia clumps, exciting at their edges HH 25 and HH 26. THRC and Rudolph and Welch (1988) proposed a similar picture for L1551 and HH 7-11, respectively. An alternative explanation to that given above is that the VLA-ammonia structure represents two independent clumps, each with its own $\mathrm{HH}$ object (HH 25 associated with the northern clump and HH 26 with the southern clump) and each with its own exciting star (the IRAS source for the northern clump and SSV 59 for the southern clump). In this interpretation, it appears that SSV 59 is the best candidate for powering the outflow, given its location closer to the centroid of the high-velocity gas. Further

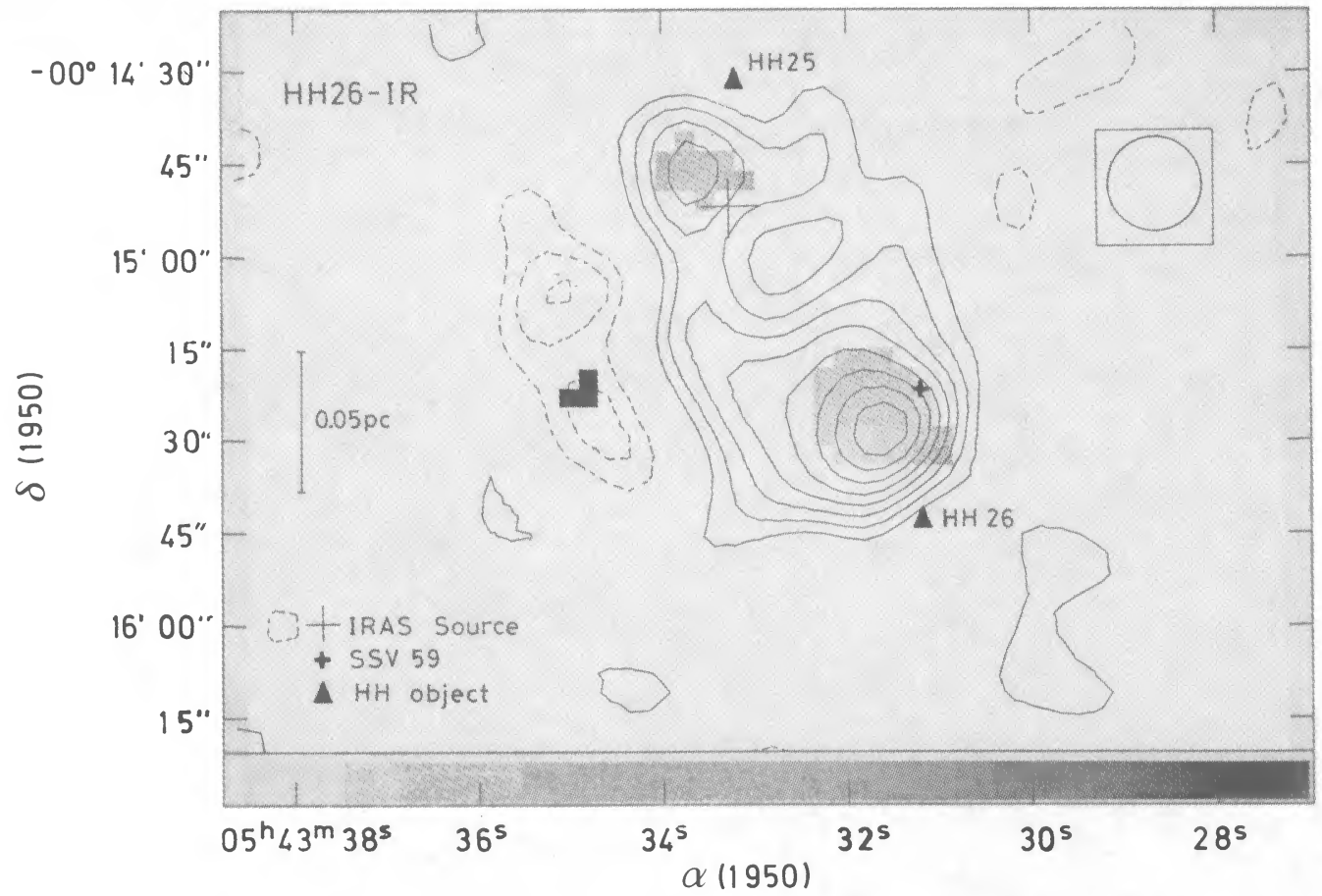

FIG. 3. $-S_{v}(1,1 ;$ is $) / S_{v}(1,1 ; \mathrm{m})$ flux ratio (gray scale) superposed on the contour map shown in Fig. 1 for HH 26 -IR. The gray scale is linear and ranges from 0.28 to 1 . A flux ratio of 0.6 is obtained at the position of both ammonia peaks. This value implies an opacity of $\tau(1,1 ; \mathrm{m}) \simeq 3$. 


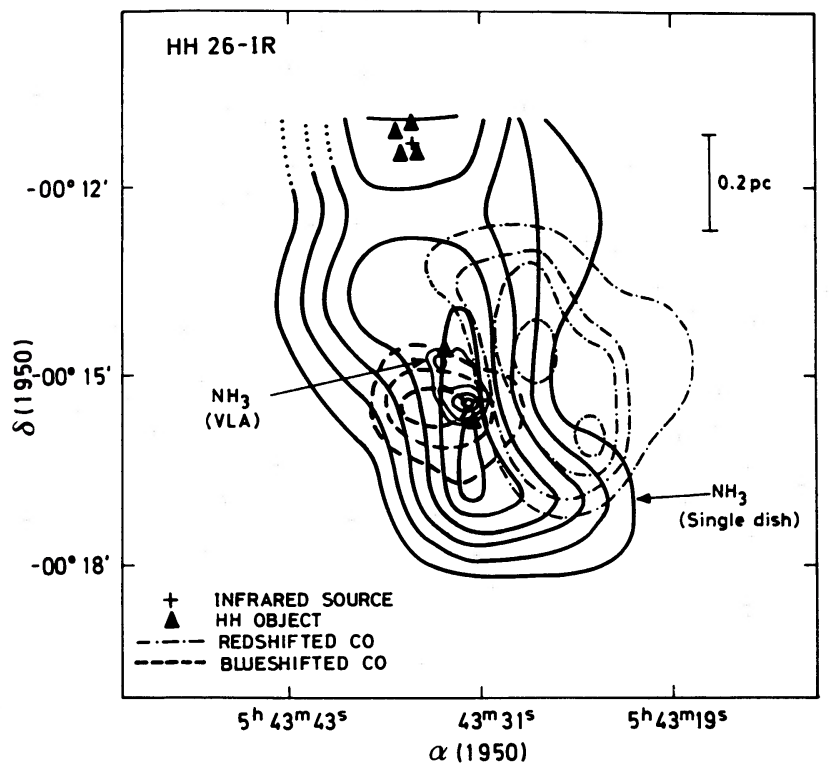

Fig. 4.-Superposition of different maps of $\mathrm{NH}_{3}$ (single dish; Torrelles et al. 1983), CO outflow (Snell and Edwards 1982), and VLA-NH 3 (this paper) for HH 26-IR.

$\mathrm{NH}_{3}$ multiline studies with higher spectral resolution to study the heating effects and the kinematics of the VLA-ammonia structure may clarify and identify the exciting source(s) in this region.

\section{b) GGD 12-15}

Our $20 \mathrm{k} \lambda$ tapered map (synthesized beam of $\simeq 9$ ") of GGD $12-15$ at $V_{\mathrm{LSR}}=12.2 \mathrm{~km} \mathrm{~s}^{-1}$ is shown in Figure 5 . As in HH 26-IR (discussed above), we differentiate two ammonia maxima with a relative position angle p.a. $\simeq 35^{\circ}$, separated by $\sim 20^{\prime \prime}$ ( $\sim 0.09 \mathrm{pc}$, at a distance of $1 \mathrm{kpc}$; Rodríguez et al. 1980), with similar peak fluxes of $\sim 160 \mathrm{mJy} \mathrm{beam}^{-1}$ and $210 \mathrm{mJy}$ beam $^{-1}$ for the northeast and southwest maxima, respectively. Given that the phase center used by THRC is located $\leq 20^{\prime \prime}$ from the two ammonia maxima, corrections by primary-beam response are not very important. The $\mathrm{H}_{2} \mathrm{O}$ maser (Rodríguez et al. 1982) and the IRS 9E source (Harvey et al. 1985) seem spatially associated with the northeast ammonia maximum. It has been proposed that these objects are related to the powering source of the $\mathrm{CO}$ bipolar outflow detected by Rodríguez et al. (1982). In Figure 6 we show a spatial velocity diagram along an axis through the two ammonia maxima. No significant velocity shift is observed with our $1.2 \mathrm{~km} \mathrm{~s}^{-1}$ spectral resolution. The low signal-to-noise ratio and the fact that the inner satellite hyperfine line at $V_{\mathrm{LSR}} \sim 19 \mathrm{~km} \mathrm{~s}^{-1}$ appears at the end of our spectral window did not allow a reliable estimate of the line opacity and hydrogen column density.

Torrelles et al. (1983), in their ammonia single-dish study (HPBW 1.4), found in GGD 12-15 an ammonia condensation with two velocity components at $V_{\text {LSR }}=11$ and $9.2 \mathrm{~km}$ $\mathrm{s}^{-1}$. We proposed that the two velocity components could be due to two different clouds interacting at an interface, producing there a young star or stars responsible for the powering of the molecular outflow. Subsequently, Güsten and Marcaide (1986), in order to define the relationship of the ammonia core and the bipolar outflow, mapped this source with HPBW $\sim 40 "$. They found an ammonia core with similar extension and morphology to those observed by Torrelles et al. (1983), but identifying three different velocity components. Interestingly, one of these components has a velocity of $V_{\mathrm{LSR}}=$ $12.1 \mathrm{~km} \mathrm{~s}^{-1}$, being located near the $\mathrm{H}_{2} \mathrm{O}$ maser and the infrared source IRS 9E with an estimated angular extension of $\leq 20^{\prime \prime} \times 20^{\prime \prime}$. We identify that velocity component with the detected VLA-ammonia structure, supporting the excellent observations of Güsten and Marcaide (1986). In Figure 7 (Plate 4) we show the overlay of our VLA-ammonia map with Figure 1 of Güsten and Marcaide (1986), which shows their single-dish ammonia map superposed on the $\mathrm{CO}$ bipolar outflow (Rodríguez et al. 1982) together with a red POSS enlargement. The VLA-ammonia structure is located at the geometrical center of the blue and redshifted $\mathrm{CO}$ lobes and is oriented perpendicular to the axis of the bipolar outflow. This result suggests that we are detecting the high-density gas directly related to the powering source of the molecular outflow. The two ammonia maxima and the perpendicular alignment with the outflow suggest that the VLA-ammonia

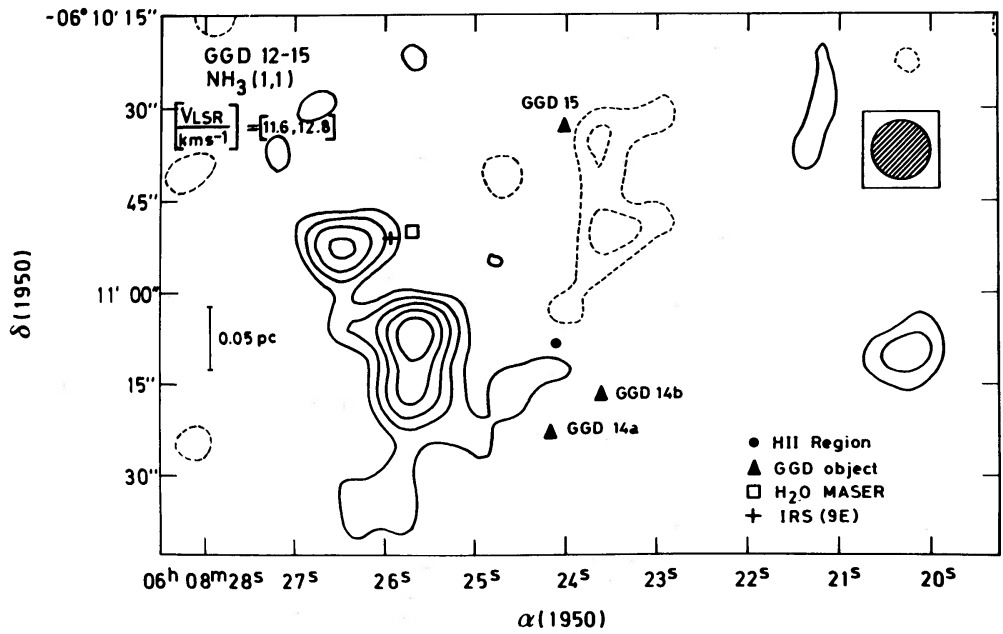

FIG. 5. - Same as Fig. 1, but for GGD 12-15. Synthesized beam size is $9 "$. The range of velocity is indicated in the figure. Contour levels are $(-4,-3,-2,2,3,4,5$, 6) $\times 30 \mathrm{mJy}_{\text {beam }}^{-1}(\mathrm{rms}=30 \mathrm{mJy})$. 


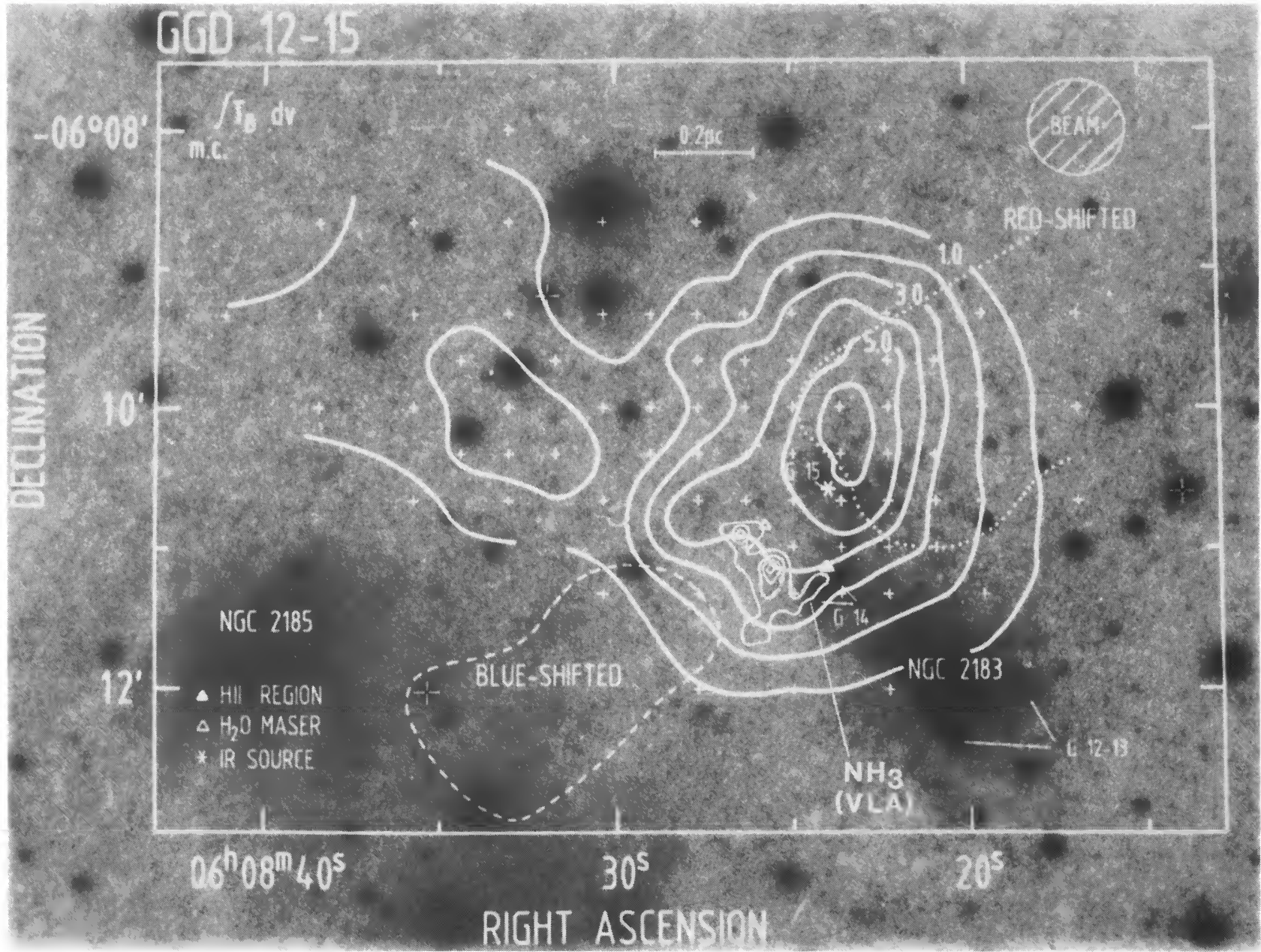

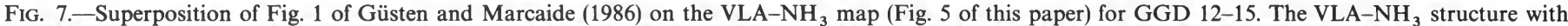
$V_{\mathrm{LSR}}=12.2 \mathrm{~km} \mathrm{~s}^{-1}$ is located at the geometrical center of the CO bipolar outflow of Rodriguez et al. (1982) with a perpendicular alignment. Little and Heaton (1989) have found an additional ammonia condensation with $V_{\mathrm{LSR}}=10.1 \mathrm{~km} \mathrm{~s}^{-1}$, probably associated with GGD 15 .

TORRELles et al. (see 346, 759) 


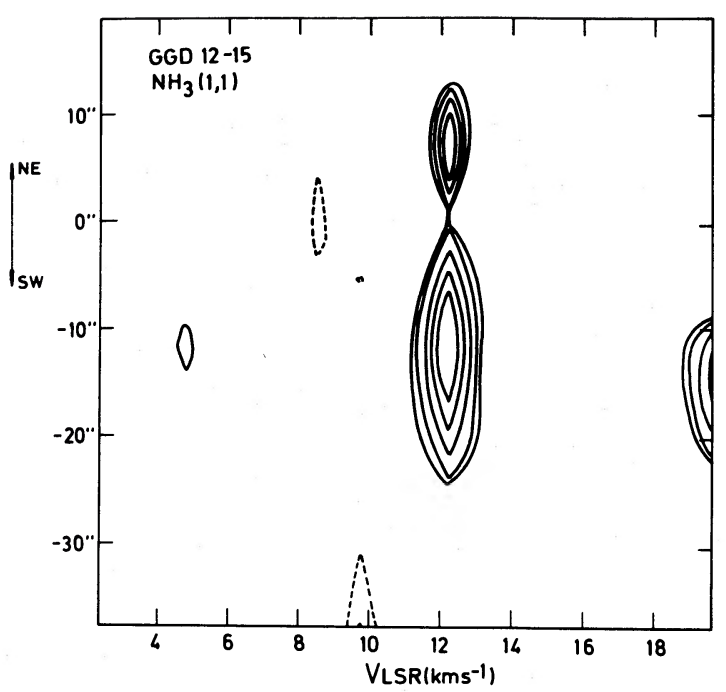

FIG. 6.- Spatial velocity diagram along an axis through the position of the two ammonia peaks of Fig. 5 (p.a. $=35^{\circ}$ ). Synthesized beam size is 9 ". Contour levels are as in Fig. 5. Spatial coordinate (vertical axis) is with respect to $\alpha(1950)=06^{\mathrm{h}} 08^{\mathrm{m}} 26^{\mathrm{s}} .2$ and $\delta(1950)=-06^{\circ} 10^{\prime} 58^{\prime \prime}$

structure could represent a molecular toroid which helps collimate the outflow. The cavity of this proposed toroid can be created by the pressure of the stellar wind powering the $\mathrm{CO}$ outflow. Again, assuming pressure equilibrium between the stellar wind and the high-density molecular gas at a radius of $0.045 \mathrm{pc}$, we obtain a hydrogen volume density of $n\left(\mathrm{H}_{2}\right) \simeq 2$ $\times 10^{5} \mathrm{~cm}^{-3}$ for a momentum rate of the stellar wind of $\dot{M} V_{*}=4 \times 10^{-4} M_{\odot} \mathrm{km} \mathrm{s}^{-1} \mathrm{yr}^{-1}$ (Rodríguez et al. 1982) and a velocity dispersion of $1.2 \mathrm{~km} \mathrm{~s}^{-1}$. This derived density is quite plausible for the excitation of $\mathrm{NH}_{3}$.

Little and Heaton (1989) have recently mapped this region with the VLA in the $(1,1)$ and $(2,2)$ lines of ammonia. They find a clumpy medium, with the brightest condensation at
$V_{\mathrm{LSR}}=12.5 \mathrm{~km} \mathrm{~s}^{-1}$ being similar to that found by us. In addition, they find at $V_{\mathrm{LSR}}=10.1 \mathrm{~km} \mathrm{~s}^{-1}$ an ammonia clump $\sim 30^{\prime \prime}$ to the northwest of the central region. This condensation is also present in our data and appears to be associated with the nebulous object GGD 15.

Heaton et al. (1988) found $\mathrm{HCO}^{+}(J=3 \rightarrow 2)$ emission perpendicular to the bipolar outflow and peaking near the $\mathbf{H}$ II $/ \mathrm{H}_{2} \mathrm{O}$ maser region. These authors conclude that the molecular gas in GGD 12-15 has several clumps with density a few times $10^{5} \mathrm{~cm}^{-3}$ embedded in a medium with lower gas density $\left[n\left(\mathrm{H}_{2}\right) \simeq 10^{3}-10^{4} \mathrm{~cm}^{-3}\right]$. The location of the VLA-ammonia structure with $V_{\mathrm{LSR}}=12.2 \mathrm{~km} \mathrm{~s}^{-1}$ and our estimated hydrogen volume density indicate that we are detecting one of the clumps predicted by Heaton et al. (1988). Higher spectral resolution observations could detect a velocity gradient as is expected in a rotating toroid. A kinetic temperature study will also help to identify the location of the exciting source.

\section{c) Serpens}

This region contains a very extended $\left(\geq 6^{\prime}\right)$ molecular outflow with an irregular morphology (Bally and Lada 1983). Single-dish ammonia observations show two peaks separated by $\sim 4^{\prime}$ (Ho and Barrett 1980; Ungerechts and Güsten 1984), with the northwest peak spatially associated with several compact $\mathrm{H}$ II regions and $\mathrm{H}_{2} \mathrm{O}$ masers (Rodríguez et al. 1980; Snell and Bally 1986). The phase center of the VLA-ammonia observations of THRC was located near that peak of ammonia emission. In Figure 8 we show our $8 \mathrm{k} \lambda$ tapered map (synthesized beam $\simeq 16^{\prime \prime}$ ) at $V_{\mathrm{LSR}}=8.2 \mathrm{~km} \mathrm{~s}^{-1}$ as a closeup of the northwest single-dish ammonia peak. VLA-ammonia emission at relatively low level $(\sim 6 \sigma)$ is detected $\sim 1.4$ north of the compact $\mathrm{H}$ in regions. This condensation is slightly elongated, with its major axis having p.a. $30^{\circ}$. No VLA-ammonia emission up to $3 \sigma$ level ( $\geq 160 \mathrm{mJy}$ ) is observed toward the compact $\mathrm{H}$ il regions.

Recently, Gómez de Castro, Eiroa, and Lenzen (1988) have

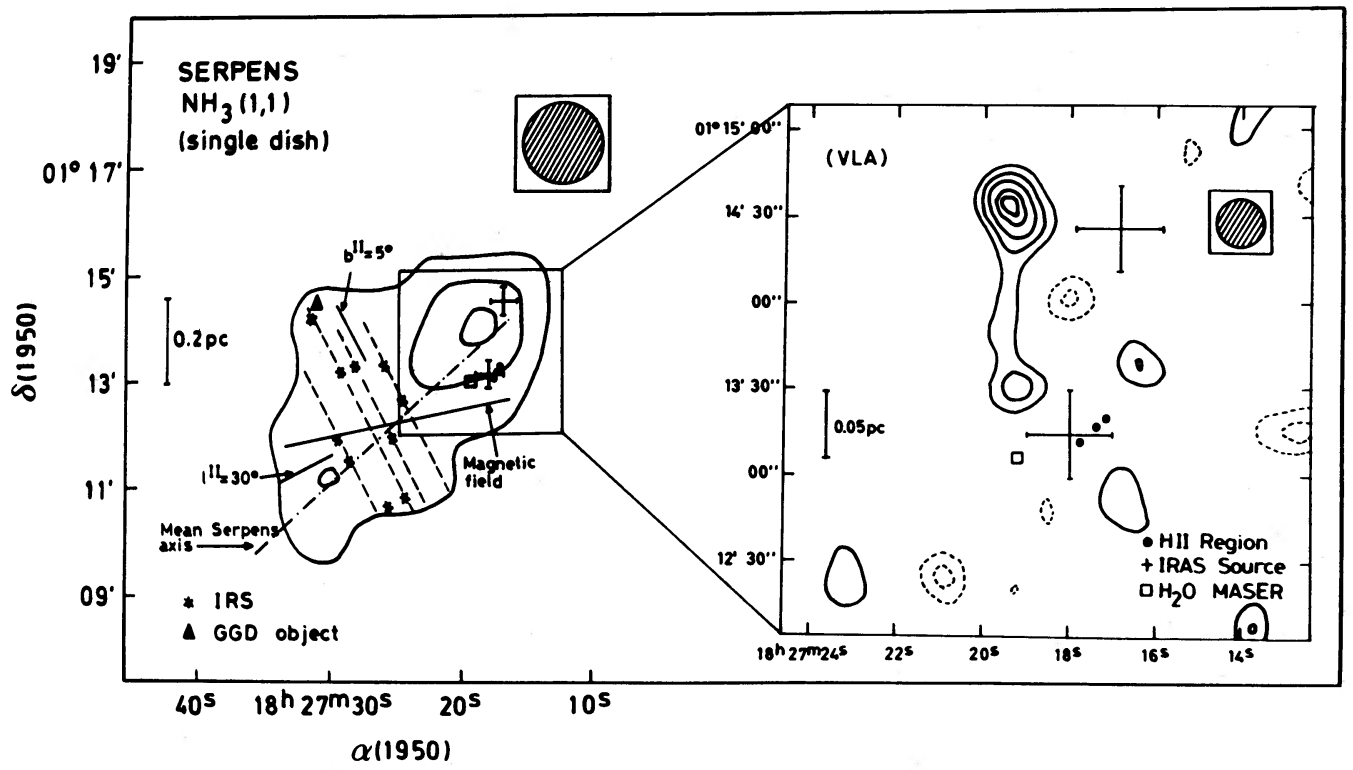

FIG. 8. - VLA contour map (synthesized beam size $\left.=16^{\prime \prime}\right)$ of the $(1,1)$ ammonia main transition in Serpens at the $\left(V_{\mathrm{LSR}} / \mathrm{km} \mathrm{s}^{-1}\right)=(7.6,8.8)$ range of velocity $($ this

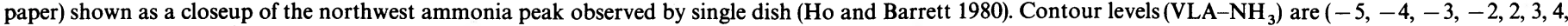
$5,5.5) \times 53 \mathrm{mJy}_{\text {beam }}^{-1}(\mathrm{rms}=53 \mathrm{mJy}$ ). We also plot Fig. 7 of Gómez de Castro, Eiroa, and Lenzen (1988), which shows the orientation of the helical component of the local magnetic field, the position of the known young Serpens objects (marked by stars), as well as the mean Serpens axis defined by the mean axis of the Serpens nebulous objects (see text). 


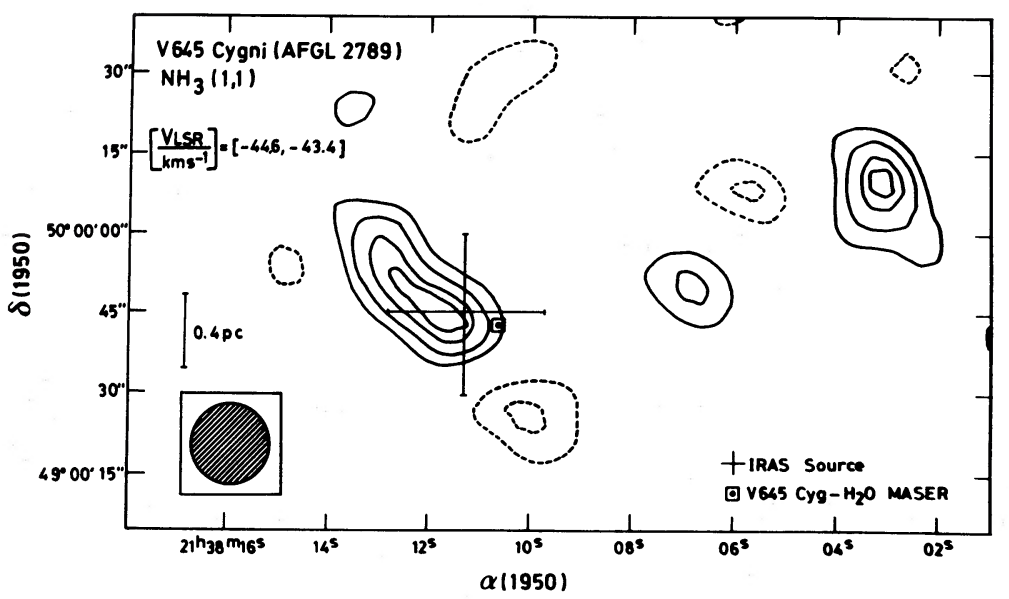

Fig. 9. - Same as Fig. 1, but for V645 Cygni (AFGL 2789). Synthesized beam size is 14". Velocity range is indicated in the figure. Contour levels are (-5, -4 , -3 , $-2,2,3,4,4.5,5) \times 38 \mathrm{mJy}$ beam ${ }^{-1}(\mathrm{rms}=38 \mathrm{mJy})$. V645 $\mathrm{Cyg}-\mathrm{H}_{2} \mathrm{O}$ maser position adopted from Lada et al. (1981).

discussed the role of the magnetic field in Serpens. They found seven nebulous objects with their major symmetry axis correlated with the helical component of the interstellar magnetic field. They speculate that several infrared sources are mainly located in layers stratified and parallel to $b=5^{\circ}$, nearly perpendicular to this component of the magnetic field (see Fig. 8). Based on these results they speculate that these stars could have been formed in a sheetlike configuration with layers of molecular gas as predicted in the model of Pudritz and Silk (1987) in the presence of a magnetic field. High angular resolution observations of molecular tracers of column density (e.g., $\mathrm{C}^{18} \mathrm{O}$ ) and high-density gas (e.g., $\left.\mathrm{NH}_{3}, \mathrm{CS}, \mathrm{HCN}\right)$ toward the layers of infrared sources seem to be important to test this possible interpretation of Gómez de Castro, Eiroa, and Lenzen (1988).

\section{d) V645 Cygni (AFGL 2789)}

Our $10 \mathrm{k} \lambda$ tapered map (synthesized beam $\simeq 14^{\prime \prime}$ ) of V645 Cygni shows two condensations at $V_{\mathrm{LSR}}=-44 \mathrm{~km} \mathrm{~s}^{-1}$ with relatively low intensity ( $\sim 5 \sigma$ or $185 \mathrm{mJy}$; Fig. 9). The western ammonia condensation is spatially unresolved. We estimate for this condensation a flux of $\sim 500 \mathrm{mJy} \mathrm{beam}^{-1}$ when primary-beam corrections are considered. No signs of star formation have been observed at present toward that condensation. The eastern condensation is resolved in the northeast-southwest direction, with the optical object V645 Cygni (AFGL 2789) also detected at the four frequency bands of IRAS. located at its edge. This displacement of the dense molecular gas could explain why this object, and its associated “duck" reflection nebula (Goodrich 1986; Lenzen 1987), are observed at optical wavelengths. The elongated VLA- $\mathrm{NH}_{3}$ structure is nearly concentric with the elongated single-dish structure mapped by Torrelles et al. (1983), having similar orientations (Fig. 10). This indicates that we are detecting the brightest part of the extended structure observed with a single dish, and that the elongated morphology exists over a large range in size scales as observed in other sources (e.g., Cepheus A ; Torrelles et al. 1985b, 1986b).

Rodríguez, Torrelles, and Moran (1981) found a CO outflow in the region, with V645 Cyg as the probable powering source. Torrelles et al. (1987) and Schultz et al. (1989), with higher angular resolution, classified it as bipolar, but with a modest

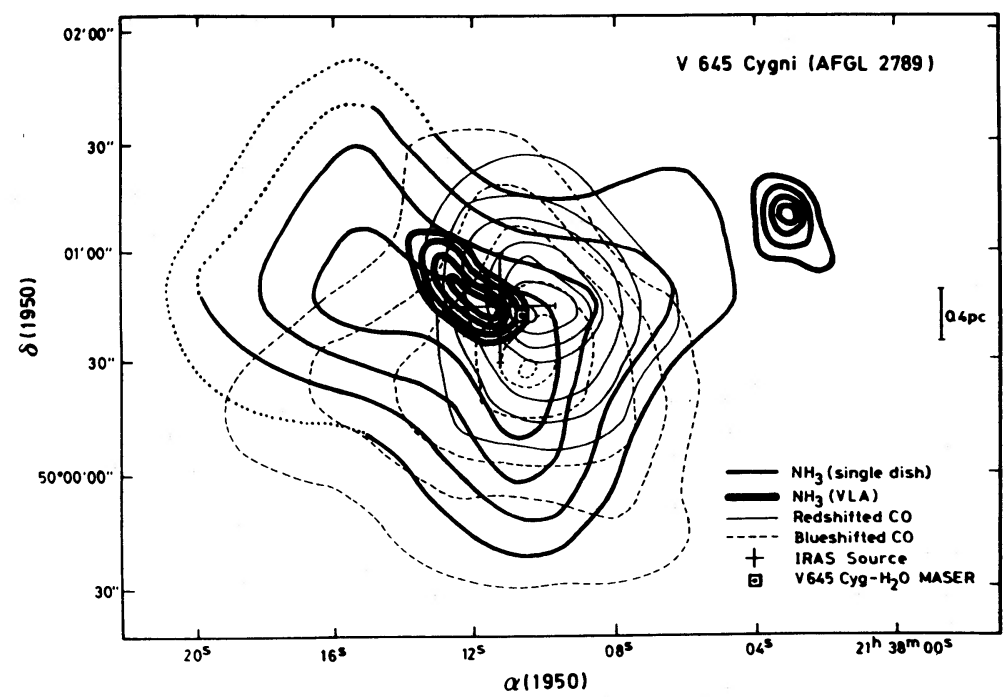

Fig. 10.-Superposition of different maps of $\mathrm{NH}_{3}$ (single dish; Torrelles et al. 1983), CO outflow (Torrelles et al. 1987), and NH 3 (VLA; this paper) for V645 Cygni. 
degree of collimation. In particular, Torrelles et al. (1987) found that the $\mathrm{CO}(J=2 \rightarrow 1)$ outflow near $\left(\leq 15^{\prime \prime}\right)$ V645 Cyg is bipolar in the north-south direction, coinciding with the direction of the optical "beak" of the Duck Nebula (see Goodrich 1986 for the description of the morphology of the optical object). However, far $\left(\geq 1^{\prime}\right)$ from V645 Cyg the bipolar outflow is reoriented into a southeast-northwest direction (Fig. 10). The bipolarity in the north-south direction near V645 Cyg has not been confirmed by the data of Schultz et al. (1988).

The VLA elongated ammonia structure cannot play an important role in the collimation processes of the $\mathrm{CO}$ outflow if V645 Cyg is its powering source. This is because of the fact that this optical source is located at the border of the VLA condensation.

\section{GENERAL COMMENTS AND CONCLUSIONS}

In the last few years a significant observational effort has been made in order to study molecular toroids with interstellar dimensions. Now we have evidence that such structures are present in several regions, e.g., Monoceros R2 (Torrelles et al. 1983, 1989b), HH 1-2 (Torrelles et al. 1985a; Marcaide et al. 1988), G35.2-0.74 (Little et al. 1985), Cepheus A (THRC; Torrelles et al. 1986b), and NGC 6334 (Jackson, Ho, and Haschick 1988). In all these sources the high-density molecular gas exhibits three defining characteristics for interstellar toroids seen nearly edge-on: (1) a high degree of elongation; (2) a velocity gradient along the major axis of the elongated highdensity molecular gas structure, which can be explained by rotating motions; and (3) stellar activity located near the kinematic center. Along these lines, two of the regions studied in this paper, HH 26-IR and GGD 12-15, are good candidates for interstellar toroids, since the high-density gas structures are elongated, with an emission double maximum, and with a perpendicular orientation with respect to the bipolar molecular outflows as expected in the theoretical models of Barral and Cantó (1981), Königl (1982), and Boss (1987). However, velocity gradients along the major axis of the $\mathrm{NH}_{3}$ structures have not been detected in these cases. We think that this lack of a velocity gradient is probably due to our low spectral resolution $\left(1.2 \mathrm{~km} \mathrm{~s}^{-1}\right)$. Higher spectral resolution observations will be useful.

We acknowledge the hospitality offered to J. M. T. by the Servicio Informático of the Universidad de Málaga (Spain) during part of the data reduction. We would like to thank Dr. L. T. Little and Dr. B. D. Heaton for a careful reading of the manuscript and helpful comments. J. M. T. and L. V. are partially supported by SEUI (Spain) grant PB87-0371 and by Junta de Andalucía (Spain).
Bally, J., and Lada, C. J. 1983, Ap. J., 265, 824

Barral, J. F., and Cantó, J. 1981, Rev. Mexicana Astr. Ap., 5, 101.

Boss, A. 1987, Ap. J., 316, 721

Clark, B. G. 1980, Astr. Ap., 89, 377

Cohen, M., and Schwartz, R. D. 1983, Ap. J. 265, 877

Genzel, R., Downes, D., Ho, P. T. P., and Bieging, J. 1982, Ap. J. (Letters), 259 L103.

Gómez de Castro, A. I., Eiroa, C., and Lenzen, R. 1988, Astr. Ap., 201, 299.

Goodrich, R. W. 1986, Ap. J., 311, 882.

Güsten, R., and Marcaide, J. M. 1986, Astr. Ap., 164, 342

Harvey, P. M., Wilking, B. A., Joy, M., and Lester, D. F. 1985, Ap. J., 288, 725

Heaton, B. D., Anderson, M., Dent, W. R. F., and Little, L. T. 1988, Astr. Ap., $203,99$.

Herbig, G. H. 1966, Vistas Astr., 8, 747

Herbst, E., and Klemperer, W. 1973, Ap. J., 185, 505

Heyer, M. C., Snell, R. L., Goldsmith, P. F., Strom, S. E., and Strom, K. M. 1986, Ap. J., 308, 134.

Ho, P. T. P., Peng, Y. L., Torrelles, J. M., Rodríguez, L. F., and Cantó, J. 1989, in preparation.

Ho, P. T. P., and Barrett, A. 1980, Ap. J., 237, 38.

Ho, P. T. P., Genzel, R., and Das, A. 1983, Ap. J., 266, 596.

Ho, P. T. P., and Townes, C. H. 1983, Ann. Rev. Astr. Ap., 21, 239.

Jackson, J., Ho, P. T. P., and Haschick, A. D. 1988, Ap. J. (Letters), 333, L73.

Königl, A. 1982, Ap. J., 261, 115.

Kwan, J., and Scoville, N. 1976, Ap. J. (Letters), 210, L39.

Lada, C. J. 1985, Ann. Rev. Astr. Ap., 23, 267.

Lada, C. J., Blitz, L., Reid, M. J., and Moran, J. M. 1981, Ap. J., 243, 769.

Lenzen, R. 1987, Astr. Ap., 173, 124.

Little, L. T., Dent, W. R. F., Heaton, B., Davies, S. R., and White, G. J. 1985, M.N.R.A.S., 217, 227.

Little, L. T., and Heaton, B. D. 1989, private communication.

Marcaide, J. M., Torrelles, J. M., Güsten, R., Menten, K. M., Ho, P. T. P.,

Moran, J. M., and Rodríguez, L. F. 1988, Astr. Ap. 197, 235.

Matthews, N., and Little, L. T. 1983, M.N.R.A.S., 205, 123

Menten, K. M., Walmsley, C. M., and Mauersberger, R. 1987, in Circumstellar Matter, ed. I. Appenzeller and C. Jordan (Dordrecht: Reidel), p. 179.

Mundt, R. 1986, Canadian J. Phys., 64, 407.

Myers, P. C., Heyer, M., Snell, R. L., and Goldsmith, P. F. 1988, Ap. J., 324, 907.

Pauls, T. A., Wilson, T. L., Bieging, J., and Martin, R. N. 1983, Astr. Ap., 124, 23.
REFERENCES

Pudritz, R. E., and Silk, J. 1987, Ap. J., 316, 213.

Rodríguez, L. F. 1988, in Galactic and Extragalactic Star Formation, ed. R. E. Pudritz and M. Fich (Dordrecht: Kluwer), p. 97.

Rodríguez, L. F., Carral, P., Ho, P. T. P., and Moran, J. M. 1982, Ap. J., 260 , 635 .

Rodríguez, L. F., Ho, P. T. P., and Moran, J. M. 1980, Ap. J. (Letters), 240, L149.

Rodríguez, L. F., Moran, J. M., Ho, P. T. P., and Gottlieb, E. W. 1980, Ap. J., 235,845 .

Rodríguez, L. F., Torrelles, J. M., and Moran, J. M. 1981, A.J., 86, 1245.

Rudolph, A. 1989, private communication.

Rudolph, A., and Welch, W. J. 1988, Ap. J. (Letters), 326, L31.

Sarcander, M., Neckel, T., and Elsässer, H. 1985, Ap. J. (Letters), 288, L51.

Schultz, A., Black, J. H., Lada, C. J., Ulich, B. L., Martin, R. N., Snell, R. L., and Erickson, N. J. 1989, Ap. J., 341, 228.

Schwartz, P. R., Waak, J. A., and Smith, H. A. 1983, Ap. J. (Letters), 267, L109.

Snell, R. L., and Bally, J. 1986, Ap. J., 303, 683.

Snell, R. L., and Edwards, S. 1982, Ap. J., 259, 668.

Snell, R. L., Loren, R. B., and Plambeck, R. L. 1980, Ap. J. (Letters), 239, L17

Spitzer, L. 1978, Physical Processes in the Interstellar Medium (New York: Wiley).

Strom, K. M., Strom, S. E., and Vrba, F. J. 1976, A.J., 81, 308.

Strom, K. M., Strom, S. E., Wolff, S. C., Morgan, J., and Wenz, M. 1986, Ap. J. Suppl., 62, 39.

Torrelles, J. M., Anglada, G., Rodríguez, L. F., Cantó, J., and Barral, J. F. 1987, Astr. Ap., 177, 171.

Torrelles, J. M., Cantó, J., Rodríguez, L. F., Ho, P. T. P., and Moran, J. M. 1985a, Ap. J. (Letters), 294, L117.

Torrelles, J. M., Ho, P. T. P., Moran, J. M., Rodríguez, L. F., and Cantó, J. $1986 a, A p . J ., 307,787$.

Torrelles, J. M., Ho, P. T. P., Rodríguez, L. F., and Cantó, J. 1985b, Ap. J., 288, 595 (THRC). . 1986b, Ap. J., 305, 721 $1989 a, A p . J ., 343,222$
$.1989 b$, Ap. J., in press.

Torrelles, J. M., Rodríguez, L. F., Cantó, J., Carral, P., Marcaide, J., Moran, J. M., and Ho, P. T. P. 1983, Ap. J., 274, 214.

Ungerechts, H., and Güsten, R. 1984, Astr. Ap., 131, 177

White, G. J., and Phillips, J. P. 1981, M.N.R.A.S., $194,947$.

Zuckerman, B., Kuiper, T. B. H., and Rodriguez Kuiper, E. N. 1976, Ap. J. (Letters), 209, L137.

J. Cantó and L. F. Rodríguez: Instituto de Astronomía, Universidad Nacional Autónoma de México, Apdo. Postal 70-264, 04510, México, D.F., México

P. T. P. Ho: Harvard-Smithsonian Center for Astrophysics, 60 Garden Street, Cambridge, MA 02138

J. M. Torrelles and Lourdes Verdes-Montenegro: Instituto de Astrofísica de Andalucía, Apdo. Correos 2144, Granada, 18080 Spain 